\section{§5. Analytic High-Beta Tokamak Equilibria with Poloidal-Sonic Flow}

Ito, A., Nakajima, N.

In magnetically confined plasmas, equilibrium flows may suppress instability and turbulent transport to give rise to improved confinement modes where high-beta is achieved. In axisymmetric toroidal systems like tokamaks, both of the poloidal and toroidal components of flow are important for these phenomena. The magnetohydrodynamic (MHD) equations for axisymmetric equilibria with flow reduce to the so-called generalized GradShafranov (GS) equation and the Bernoulli law with five free functions of the magnetic flux in axisymmetric systems. Recently, a new reduced set of equilibrium equations has been derived for high-beta tokamaks with flow in the order of the poloidal-sound velocity ${ }^{11}$. This set of equations includes the effect of poloidal flow on both the magnetic flux and the pressure in higher-order terms of asymptotic expansions in terms of the inverse aspect ratio. We have found analytical solutions for this reduced set of equations with linear profiles of the lowest order free functions, where the leading order poloidal Mach number is constant, and performed qualitative studies of equilibria with sub- and super-poloidal-sonic flow ${ }^{2}$. This is a new class of analytic equilibria that indicates both of the modification of the magnetic structure and the departure of the pressure surfaces from the magnetic surfaces by sub- or super-poloidal-sonic flows. The analytical representation for the shift of the magnetic axis from the geometrical axis and that of the pressure maximum, and the equilibrium beta limit are also obtained. We have shown that the shift of the magnetic axis from the geometric axis is enhanced by a slightly super-poloidal-sonic flow and it produces a forbidden region of equilibrium by the poloidal-sonic flow (Fig.1 (a)). Figure 1 (b) shows the magnetic structure for the poloidal Mach number $M_{A p c}^{2} / \gamma p_{1 c}=1.05$, where the equilibrium beta limit is violated since the separatrix appears in the plasma region. Figure 2 shows the pressure surfaces (black) for (a) sub-poloidal-sonic and (b) super-poloidal-sonic flows in comparison with their magnetic surfaces (gray). The pressure maximum is shifted outwards for the sub-poloidal-sonic flow and inwards for the super-poloidal-sonic flow. The physical mechanism of this shift can be explained with the formalism of Ref. 1 in analogy to those of the geodesic acoustic mode and the slow magnetosonic wave. The uniform the poloidal Mach number has been assumed to for this equilibrium analysis.

Analytical representations of equilibria with flow may be useful for benchmarks for the numerical analysis of non-reduced equilibria as well as for qualitative studies of equilibrium flows and stability analyses.
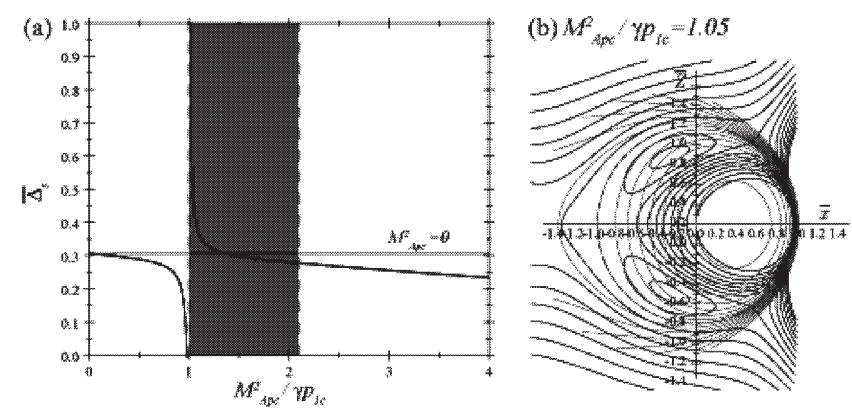

Fig.1 (a) The shift of the magnetic axis from the geometrical axis as a function of the poloidal Mach number. (b) The magnetic surfaces for the equilibrium with the poloidal Mach number 1.05 and for the static one.
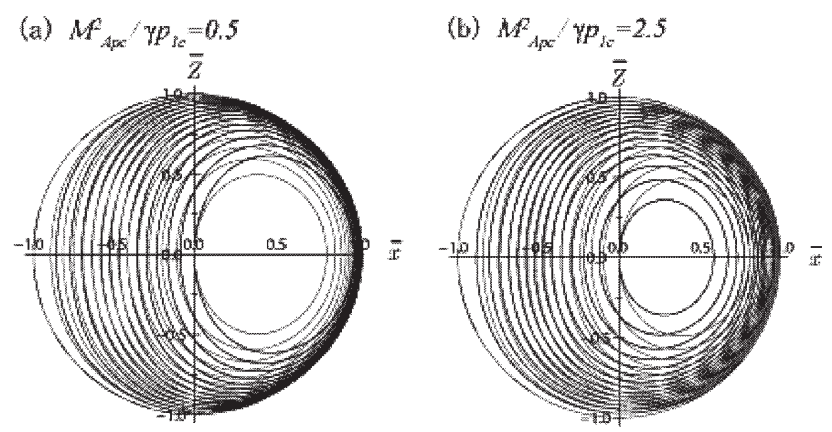

Fig.2 The pressure surfaces (black) and the magnetic surfaces (gray) for (a) sub-poloidal-sonic and (b) super-poloidal-sonic flows.

1) Ito, A., Ramos, J. J., and Nakajima, N.: Plasma Fusion Res. 3, 034 (2008).

2) Ito, A. and Nakajima, N.: Plasma Phys. Control. Fusion 51, 035007 (2009). 